ISSN: 0210-1696

DOI: http://dx.doi.org/10.14201/scero201613754

\title{
EVALUACIÓN DE LA METODOLOGÍA EMPLEO CON APOYO (ECA) EN EL PROGRAMA PROMENTOR (UAM-PRODIS). EL AJUSTE COMPETENCIAL
}

\section{Evaluation of the Methodology Supported Employment in Promentor Program (UAM-PRODIS). Competence Setting}

\author{
Dolores IZUZQUiza GasseT \\ Universidad Autónoma de Madrid
}

Pablo Rodríguez Herrero

Universidad Autónoma de Madrid y Fundación Prodis. Bulevar Indalecio Prieto, 2. CP 28032 pablorodriguez@fundacionprodis.org.

Recepción: 3 de noviembre de 2015

Fecha de aceptación definitiva: 25 de enero de 2016

RESUMEN: En este artículo se presentan los resultados de una investigación sobre el ajuste entre el puesto de trabajo y las competencias del trabajador con discapacidad intelectual, con el propósito de identificar los factores que afectan al mantenimiento del puesto de trabajo. Se lleva a cabo a través de una metodología de evaluación de resultados del Programa Promentor (UAM-PRODIS) y del Servicio de Inclusión Laboral asociado. La evaluación se ha realizado sobre 52 trabajadores, con la aplicación de un cuestionario dirigido a las empresas en las que trabajan y a sus preparadores o mediadores laborales. Los resultados más significativos son: 1) La figura del mediador laboral es esencial no solo en la inserción laboral, sino también en el mantenimiento en el puesto de trabajo; 2) La dimensión competencial más valorada es "Responsabilidad, autonomía y motivación”; 3) En la segunda dimensión competencial más valorada, hay discrepancias entre los mediadores laborales y las empresas colaboradoras. Los primeros destacan la dimensión "Competencias interpersonales", mientras que las empresas 
EVALUACIÓN DE LA METODOLOGÍA EMPLEO CON APOYO (ECA)

EN EL PROGRAMA PROMENTOR (UAM-PRODIS). EL AJUSTE COMPETENCIAL

DOLORES IZUZQUIZA GASSET Y PABLO RODRÍGUEZ HERRERO

dan más importancia a "Gestión y organización”. En las conclusiones se reflexiona sobre la implicación de estos resultados en los itinerarios de formación y apoyo laboral personalizados.

Palabras Clave: discapacidad intelectual; Empleo con Apoyo (ECA); ajuste competencial; Programa Promentor; inclusión laboral.

AвSTRACT: In this article are presented the results of research on the fit between the job and the skills of workers with intellectual disabilities, in order to identify factors that affect the maintenance of the workplace. It is done through a methodology for evaluating program outcomes Promentor (UAM-PRODIS) and associated Labor Inclusion Service. The evaluation was carried out on 52 workers, with the application of a questionnaire to the companies in which they work and their trainers or labor mediators. The most significant results are: 1) The figure of labor mediator is essential not only in employment but also in maintenance in the workplace; 2) The most valued competence dimension is "Responsibility, autonomy and motivation"; 3) In the second most valued competence dimension, there are discrepancies between labor mediators and collaborators. The first highlights the dimension "Interpersonal skills", while companies put more emphasis on "Management and organization". The conclusions reflect on the implication of these results on the itineraries of training and employment support custom.

KEY WORDS: intellectual disabilities; supported employment; competence setting; Promentor Program; labor inclusion.

\section{Introducción}

4 N UNA SOCIEDAD DEMOCRÁTICA y que impulse la participación ciudadana, la inclusión laboral es uno de los ejes fundamentales. El trabajo de calidad, ajustado a la formación, las competencias y los intereses, dignifica al trabajador e influye en el resto de dimensiones de su vida. En el caso de personas en riesgo de exclusión social, como pueden ser personas con discapacidad intelectual, la inclusión laboral adquiere si cabe mayor importancia.

Siguiendo la definición del último manual de la American Association on Intellectual and Developmental Disabilities (AAIDD) (Schalock y cols., 2010), la discapacidad intelectual es aquella caracterizada por limitaciones significativas en el funcionamiento intelectual y la conducta adaptativa manifestada en habilidades prácticas, sociales y conceptuales. Estas características requieren un sistema de apoyos que fomente la inclusión de las personas con discapacidad intelectual.

En el ámbito laboral, la inserción en empresas ordinarias se realiza a través de la metodología de Empleo con Apoyo (ECA). Ésta asegura que el trabajador con discapacidad intelectual y la empresa que contrata tengan un apoyo en la figura del mediador o preparador laboral a lo largo de todo el proceso de inserción laboral.

En el desarrollo de programas de ECA, las organizaciones de apoyo a personas con discapacidad intelectual ejercen una función esencial. La Fundación Prodis, en 
colaboración con la Universidad Autónoma de Madrid (España, UAM), tiene un Servicio de Empleo con Apoyo asociado al Programa Promentor, en el marco del cual se desarrolla el Título Propio "Formación para la Inclusión Laboral de Jóvenes con Discapacidad Intelectual”.

Estos programas se someten, para su mejora, a un análisis permanente de su funcionamiento. La evaluación constituye una práctica imprescindible en toda actividad planificada, ya que es la fase que ofrece información continua sobre el grado de cumplimiento de los objetivos intermedios de las organizaciones y, en última instancia, de los resultados que se alcanzan. De esta forma, la mejora de los proyectos y de las intervenciones se basa en evidencias resultantes de los informes de investigación (Substance Abuse and Mental Health Services Administration, 2009).

En este artículo se presenta una investigación que evalúa el ajuste competencial entre el trabajador con discapacidad intelectual y el puesto de trabajo. El objetivo es identificar factores que influyen en la inserción laboral y en el mantenimiento del puesto de trabajo.

El ECA es una metodología de apoyo para la inclusión laboral que surge en los años 80 del siglo pasado en EE. UU. Uno de sus padres, P. Wehman, lo definía como (Wehman y cols., 1987) "un empleo competitivo en entornos integrados, para aquellos individuos que tradicionalmente no han tenido esta oportunidad, utilizando entrenadores laborales preparados adecuadamente y fomentando la formación sistemática, el desarrollo laboral y los servicios de seguimiento entre otros” (p. 180). En España se daría a conocer en el I Symposium Internacional sobre ECA, organizado por el Consejo Insular de Mallorca y la Universidad de las Islas Baleares (Esteban y Jordán de Urríes, 2006). El ECA ha evolucionado hasta extenderse geográficamente en la sociedad occidental, y se ha abierto a los distintos tipos de discapacidad y a otras posibles causas de exclusión social (Jordán de Urríes, Beyer y Verdugo, 2008).

El ECA tiene las siguientes características principales (Jordán de Urríes, 2010):

- Empleo: El propósito de estos programas es el empleo con todos los resultados normales derivados de obtenerlo. Sueldo, condiciones de trabajo y seguridad del empleo son consideraciones clave.

- Apoyo continuado: El objetivo es proporcionar el apoyo continuado requerido para alcanzar y mantener un empleo en vez de tener una persona preparada para un trabajo que pueda llegar en un futuro.

- Empleos, no servicios: El énfasis se pone en crear oportunidades de empleo en vez de proporcionar solamente servicios para desarrollar habilidades.

- Participación plena: Las personas con discapacidades severas no son excluidas. Se asume que todas las personas, independientemente del nivel de su discapacidad, tienen la capacidad de acogerse al empleo con apoyo si pueden proporcionarse los servicios de apoyo continuado necesarios.

- Variedad y flexibilidad: El empleo con apoyo no cierra los programas en una o dos opciones de trabajo. Es flexible debido al amplio rango de empleos en la 
EVALUACIÓN DE LA METODOLOGÍA EMPLEO CON APOYO (ECA)

EN EL PROGRAMA PROMENTOR (UAM-PRODIS). EL AJUSTE COMPETENCIAL

DOLORES IZUZQUIZA GASSET Y PABLO RODRÍGUEZ HERRERO

comunidad y a las diferentes maneras de proporcionar apoyo a las personas en esos empleos.

En cuanto a las fases para su desarrollo, la European Union of Supported Employment (EUSE) propone las siguientes (Evans y cols., 2005):

1. Introducción al servicio de empleo con apoyo.

2. Trazado de un perfil profesional.

3. Búsqueda de empleo.

4. Implicación de la empresa.

5. Dotación de apoyos dentro y fuera del lugar de trabajo.

Estas etapas definen unos criterios de calidad de los procesos de inserción laboral a través del ECA. Es importante, en este sentido, seguir los procedimientos establecidos por la metodología (Becker y cols., 2006). Para establecer estándares de calidad, se han creado distintos instrumentos de evaluación, entre los que destacan la Supported Employment Fidelity Scale (Bond y cols., 2012) o el Sistema de Evaluación Multicomponente de Programas de Empleo con Apoyo (Verdugo, Jordán de Urríes y Vicent, 2009).

Estos estándares orientan los planteamientos y el desarrollo de los programas de ECA, al mismo tiempo que son compatibles con propuestas de mejora fundamentadas en las evidencias resultantes en las investigaciones evaluativas. El amplio informe de la Comisión Europea (2011), a través del estudio del ECA en 30 países, destaca la figura del mediador laboral como una parte esencial de la inserción laboral. El mediador laboral es "el principal valedor de la persona con discapacidad y el encargado de diseñar, junto con la propia persona, los diferentes itinerarios laborales” (Arenas y González, 2010: 47). Por tanto, el mediador laboral es un apoyo fundamental tanto para la empresa como para el trabajador con discapacidad a lo largo de toda su vida laboral (Egido, Cerrillo y Camina, 2009; Izuzquiza y Herrán, 2010).

Otras investigaciones evaluativas se centran en la importancia de involucrar a la familia en la transición al empleo (Foley y cols., 2014; Hall y cols., 2014; West y Patton, 2010), en la calidad de vida percibida por los trabajadores con discapacidad intelectual (Flores y cols., 2011; Santamaría y cols., 2012; Verdugo y cols., 2009; Verdugo y cols., 2012), en metodologías de apoyo en el entorno laboral (Bennett y cols., 2010; Goh y Bambara, 2013; O’Brien y Callahan, 2010) o en la percepción de los empleadores acerca del ECA. En un estudio realizado en Escocia (Coutts y Riddell, 2013), los empleadores de personas con discapacidad manifestaron que, en general, su inserción laboral tiene un impacto positivo en las empresas. Asimismo, percibían que era muy importante el ajuste entre el puesto de trabajo y las competencias e intereses del trabajador. La investigación que se presenta en este artículo aborda este último aspecto, por su interés tanto para una inserción laboral positiva como para identificar factores que influyen en el mantenimiento del puesto de trabajo en el tiempo. 
EVALUACIÓN DE LA METODOLOGÍA EMPLEO CON APOYO (ECA)

EN EL PROGRAMA PROMENTOR (UAM-PRODIS). EL AJUSTE COMPETENCIAL

DOLORES IZUZQUIZA GASSET Y PABLO RODRÍGUEZ HERRERO

El Programa Promentor: Título Propio en la Universidad Autónoma de Madrid y Servicio de Inclusión Laboral

La investigación se realiza en el marco del Programa Promentor y de la Cátedra de Patrocinio UAM-PRODIS. El Programa Promentor es el primer título de formación universitaria para personas con discapacidad intelectual en España. Nació en 2004 por iniciativa de la Fundación Prodis, en el Departamento de Didáctica y Teoría de la Educación de la Universidad Autónoma de Madrid (Izuzquiza, 2012). El curso que realizan los alumnos se ha reconocido por la universidad como un “Título Propio en Formación para la Inclusión Laboral de Jóvenes con Discapacidad Intelectual".

El curso se desarrolla a lo largo de dos años académicos en un entorno universitario inclusivo, y aporta una formación en competencias profesionales para la inserción laboral, así como la enseñanza de habilidades sociales y emocionales para la mejora de la calidad de vida (Cerrillo, Izuzquiza y Egido, 2013). Hasta la fecha, más de 100 jóvenes con discapacidad intelectual han estudiado el título propio, con un índice de empleo posterior a los estudios del $92 \%$.

Al finalizar los estudios universitarios, la Fundación Prodis, a través del Servicio de Inclusión Laboral, apoya a los titulados en la inserción laboral a través de la metodología de ECA. La Cátedra de Patrocinio UAM-PRODIS ha desarrollado varias investigaciones cuyos objetivos han sido validar el plan de estudios del Programa Promentor y estudiar su relación con el desarrollo de competencias laborales para la inclusión en empresas ordinarias.

Estas investigaciones ponen de manifiesto la importancia de la formación laboral y de la figura del mediador laboral en el proceso de inclusión laboral de personas con discapacidad intelectual. El estudio que se presenta en este artículo responde a la necesidad de hacer una investigación más amplia del ajuste competencial de los trabajadores y de los factores que inciden en el mantenimiento en los puestos de trabajo.

\section{Metodología}

\section{Participantes}

En la investigación se ha realizado un análisis del ajuste competencial de 52 trabajadores con discapacidad intelectual. Para la valoración del ajuste competencial se ha aplicado un cuestionario dirigido a responsables o compañeros del trabajador en la empresa, que han contestado 62 personas (en el caso de algunos trabajadores con discapacidad intelectual, el ajuste competencial fue evaluado por varios profesionales de su empresa). Con el objetivo de hacer un análisis comparativo, el cuestionario también lo han contestado 6 mediadores laborales de los trabajadores.

Prácticamente la mitad de los informantes de las empresas son apoyos naturales (47.5\%), seguidos por la categoría jefe, superior o mando directo $(15.3 \%)$ : 
EVALUACIÓN DE LA METODOLOGÍA EMPLEO CON APOYO (ECA)

EN EL PROGRAMA PROMENTOR (UAM-PRODIS). EL AJUSTE COMPETENCIAL

DOLORES IZUZQUIZA GASSET Y PABLO RODRÍGUEZ HERRERO

\section{FIGURA 1. Relación entre el informante y el trabajador con DI}

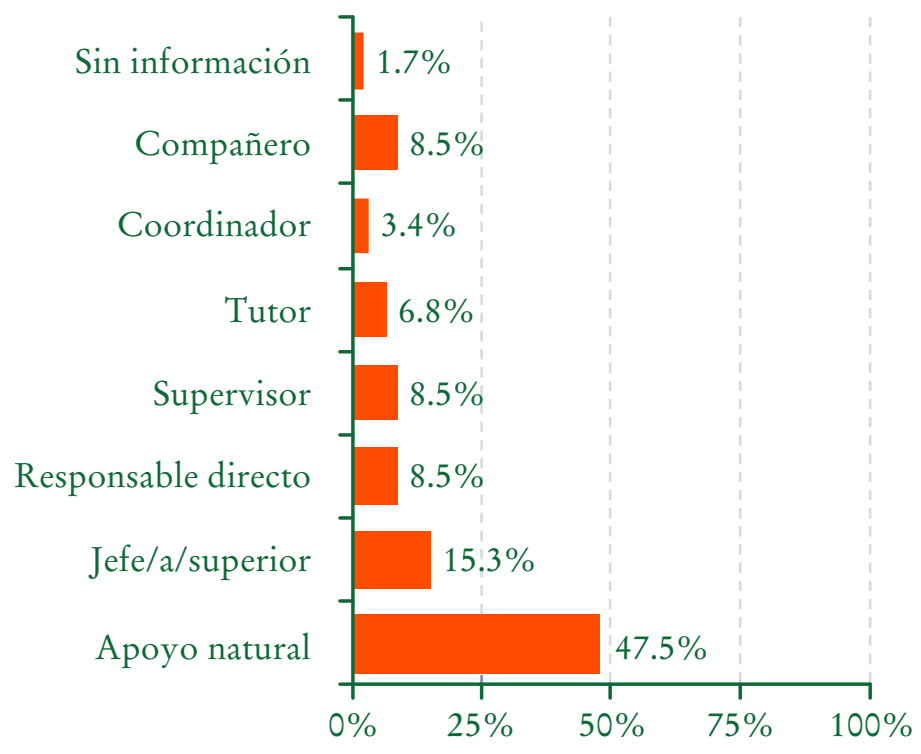

Fuente: Elaboración propia.

La edad media de los trabajadores es de 27 años, con edades comprendidas entre los 22 y los 40 años. El 51.9\% de los trabajadores son hombres (edad media 26.6 años) y el $48.1 \%$ son mujeres (edad media 27.5 años).

El nivel de estudios de los trabajadores participantes es bastante heterogéneo, predominando los estudios de la Educación Secundaria Obligatoria (ESO) $(32.7 \%)$ y de Transición a la vida Adulta (TVA) (30.6\%).

\section{Objetivos}

El objetivo de la presente investigación es identificar los principales factores de éxito (y de riesgo) que inciden directa o indirectamente en el mantenimiento o permanencia en el puesto de trabajo de las personas con discapacidad intelectual.

Concretamente, se definen los siguientes objetivos específicos:

- Evaluar el ajuste competencial de los trabajadores con discapacidad intelectual en empresas colaboradoras con Promentor, la adaptación de los puestos y el nivel de desempeño exigido por los empresarios.

- Identificar factores que influyen de forma positiva en la sostenibilidad del trabajador en el puesto a medio y largo plazo, así como aspectos a mejorar en la mediación laboral. 
- Valorar, con las entidades anteriores, la relación entre mantenimiento en el puesto y las siguientes dimensiones competenciales del trabajador con discapacidad intelectual: Gestión y organización del tiempo; Apariencia personal e higiene; Flexibilidad, control emocional y resistencia a la frustración; Responsabilidad, autonomía y motivación; Competencias interpersonales y Competencias comunicativas.

- Valorar el papel que juega el mediador laboral en el mantenimiento del puesto y frecuencia óptima de las intervenciones.

- Relacionar el grado de autonomía personal con el mantenimiento en el puesto o, en su caso, con el aumento de la empleabilidad.

Este estudio forma parte de una investigación más amplia. En un estudio posterior, se profundizará en la identificación de los cambios de carácter humano y organizativo que produce la incorporación y el mantenimiento en el puesto de trabajo de personas con discapacidad intelectual en las empresas.

\section{Diseño de la evaluación}

El presente trabajo puede definirse como una evaluación de resultados (Bisquerra, 2004). La evaluación de resultados permite obtener informaciones y analizar el cumplimiento de las propuestas para los objetivos específicos del programa en términos de su actividad, eficacia y eficiencia para obtener conclusiones que permitan retroalimentar la gestión del programa, además de identificar, planificar y gestionar otros programas y proyectos. La investigación evaluativa se sitúa, por consiguiente, en un diseño cuyo objetivo es la mejora del programa (Stufflebeam, 2003).

Esta evaluación concluye con un informe que permite identificar la efectividad, la validez y la eficiencia del programa, así como obtener conclusiones y recomendaciones a futuro tanto del programa evaluado como de otros programas o proyectos.

Es de destacar que el proyecto de investigación ha tenido como uno de sus principios fundamentales el respeto ético por los participantes en todos sus niveles, siguiendo los criterios de la World Medical Association Declaration of Helsinki (2002). Asimismo, se ha sometido a la aprobación del Comité de Ética de la Fundación Prodis.

El proceso de evaluación se inició en el último trimestre de 2013, presentándose los informes pertinentes en los primeros meses de 2015.

\section{Procedimiento y estrategias de recogida de información}

El instrumento principal de la investigación es un cuestionario dirigido a profesionales de las empresas en las que trabajan las personas con discapacidad intelectual participantes en el estudio y a mediadores laborales. El cuestionario que se utilizó se 
creó y validó previamente en un trabajo de investigación (Pérez e Izuzquiza, 2011). El cuestionario incluye las siguientes dimensiones de análisis:

- Gestión y organización del tiempo.

- Apariencia personal e higiene.

- Flexibilidad, control emocional y resistencia a la frustración.

- Responsabilidad, autonomía y motivación.

- Competencias interpersonales.

- Competencias comunicativas.

La definición de estas dimensiones, en la construcción y validación del cuestionario, se ha sometido a un análisis de juicio de expertos, en el que han participado activamente el equipo de mediadores laborales del Servicio de Inclusión Laboral. Este análisis ha posibilitado el cambio en el planteamiento de algunos ítems, la supresión o la inclusión de otros que en la primera versión del cuestionario no se introducían. En este sentido, se introdujo un ítem fuera de las dimensiones competenciales descritas, relativo a "Mantener el ritmo y la carga de trabajo adecuadamente", y otros sobre la orientación en la empresa. Por otra parte, la tipología de preguntas es variada, incluyéndose cuestiones abiertas, cerradas con opciones de respuesta definidas o de clasificación por grado de desempeño o importancia.

El cuestionario incluye una primera parte de datos generales (nombre, fecha de nacimiento, puesto que ocupa el trabajador en la empresa, tiempo que lleva en la empresa, tipo de contrato, procedencia del trabajador y nivel de estudios), ítems sobre la adaptación del puesto de trabajo (tipología del puesto de trabajo, creación del puesto de trabajo, adaptaciones realizadas, etc.) y dos cuestiones relativas al ajuste competencial:

- Definir qué dimensiones competenciales (de las descritas anteriormente) tienen más importancia para que un trabajador mantenga su puesto de trabajo, ordenándolas de mayor a menor importancia. En este apartado se incluyó la valoración para tres poblaciones distintas: trabajadores sin discapacidad intelectual, trabajadores con discapacidad intelectual y trabajador con discapacidad intelectual de la empresa.

- Valoración de la consecución de las competencias descritas del trabajador con discapacidad intelectual que trabaja en la empresa y grado de importancia que tienen dichas competencias para el desempeño de su puesto de trabajo. Cada dimensión incluye una serie de ítems, con un total de 43.

El ajuste competencial se mide comparando el nivel de competencia del trabajador en cada ítem con la valoración de la importancia que se le da a dichas competencias en el desempeño del puesto de trabajo. El cuestionario lo contestaron tanto los mediadores laborales como las empresas colaboradoras (jefes, apoyos naturales, etc.). 
EVALUACIÓN DE LA METODOLOGÍA EMPLEO CON APOYO (ECA)

EN EL PROGRAMA PROMENTOR (UAM-PRODIS). EL AJUSTE COMPETENCIAL

DOLORES IZUZQUIZA GASSET Y PABLO RODRÍGUEZ HERRERO

Plan de análisis

Los resultados del análisis se agrupan en tres bloques:

1. Análisis descriptivo de los participantes, agentes y adaptación del puesto.

2. Construcción de rankings de importancia respecto a la permanencia en el puesto de trabajo. En este bloque se comparan los rankings establecidos por los mediadores laborales y los resultantes de las empresas colaboradoras.

3. Análisis estadístico de tipo descriptivo y comparativo de cada uno de los 43 ítems que incluyen las dimensiones competenciales.

\section{Resultados}

Participantes, agentes y adaptación del puesto

En este apartado se hace un análisis descriptivo de datos relativos a los trabajadores, a las empresas o a las adaptaciones realizadas en los puestos de trabajo.

El tipo de contratación más frecuente es la contratación fija, con un $66.7 \%$ de casos, seguida de la temporal con un $31.4 \%$. Uno de los trabajadores participantes tiene contrato en prácticas. Por otro lado, predominan los participantes que llevan más tiempo en la empresa, con un $32.7 \%$ de trabajadores que llevan más de 4 años, frente a un $17.3 \%$ que llevan menos de 1 año.

El puesto desempeñado por los trabajadores participantes se concentra especialmente en las categorías "Auxiliar administrativo" (38.5\%) y "Ayudante de auxiliar administrativo” (17.3\%). Las empresas colaboradoras con la investigación destacan por su heterogeneidad: están presentes desde bancos y aseguradoras importantes por su volumen a empresas compuestas por menos de 10 trabajadores, así como sectores de actividad diversos. La mayoría de puestos (84.6\%) son de carácter permanente. El 63.5\% de los puestos se crearon cuando se incorporó el trabajador con discapacidad intelectual a la empresa, frente al 30.8\% que eran puestos que ya existían anteriormente (5.8\% NS/NC). El 23.6\% de los puestos existen desde hace 2 años o menos, mientras que un 23.5\% existen desde hace 2-4 años. Un $27.5 \%$ de los puestos existen desde hace entre 5 y 10 años y un $15.7 \%$ desde hace más de 10 años.

Existe un amplia mayoría de puestos analizados que han sido adaptados para el trabajador con discapacidad intelectual (88.5\%). Solamente hubo 4 casos en los que no fue necesario realizar una adaptación específica. Entre los puestos identificados como no adaptados, en un caso se argumentó que el "trabajador pudo desempeñarlo sin obstáculos”, en otro caso que el puesto "ya existía” y en otro que el puesto "se creó expresamente para el trabajador" (no se obtuvo información sobre el cuarto puesto no adaptado). La dificultad que conllevó la adaptación de los puestos analizados es percibida por las empresas colaboradoras como moderada o baja, con un 
43.6\% de respuestas situadas en los valores medios de la escala o "dificultad media", y un $38.2 \%$ de respuestas situadas en los valores de menor dificultad.

En cuanto a las dificultades que detectan las empresas colaboradoras en la adaptación de los puestos de trabajo, los informantes describen hasta un total de 62 dificultades que ha de afrontar la mediación laboral. En cualquier caso, la valoración de la actividad desarrollada por los mediadores laborales es muy alta, alcanzando un valor promedio de 6,7 sobre 7 .

La figura del mediador laboral no solo es necesaria al inicio del proceso de inserción laboral, como lo demuestra que más del $90 \%$ de los informantes encuestados considere importante que continúe realizando visitas a lo largo de la vida laboral del trabajador con el fin de mantener el desempeño en el puesto de trabajo. En cualquier caso, la mayoría de las empresas colaboradoras $(91.9 \%)$ consideran que, una vez que el trabajador es autónomo en las tareas encomendadas, el mediador laboral debería ir 1 vez al mes.

\section{Dimensiones subyacentes a la permanencia en el puesto}

En el caso de los mediadores laborales de los trabajadores con discapacidad intelectual, la dimensión competencial que consideran más importante para lograr

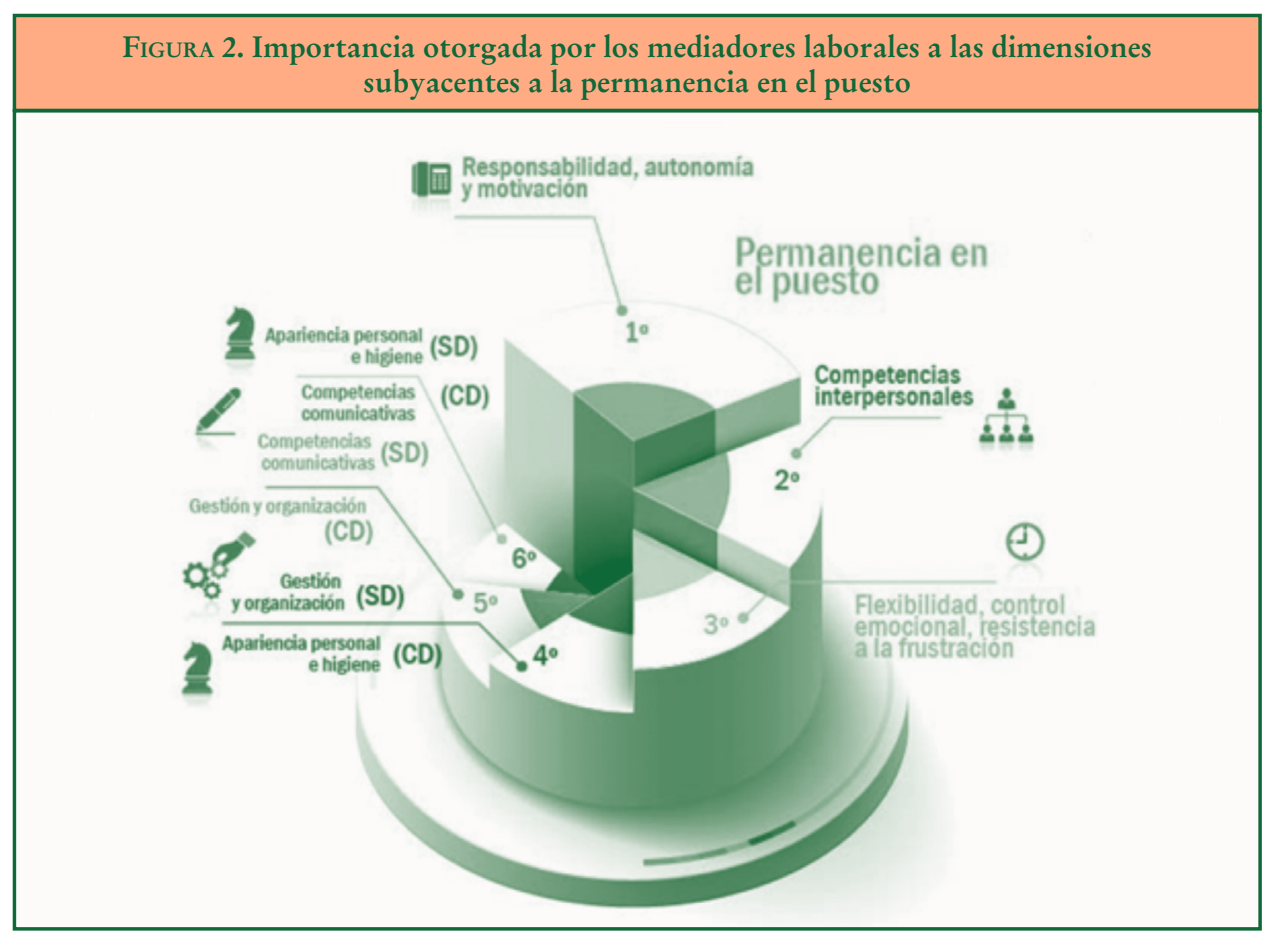

Fuente: Elaboración propia.

(C) Ediciones Universidad de Salamanca / CC BY-NC-ND

Siglo Cero, vol. 47 (1), n. $.^{\circ} 257,2016$, enero-marzo, pp. 37-54 
mantener el puesto de trabajo es "Responsabilidad, autonomía y motivación”, seguida de las dimensiones "Competencias interpersonales" y "Flexibilidad, control emocional y resistencia a la frustración” (Figura 2).

En cuanto a la comparación de los resultados con trabajadores sin discapacidad intelectual, no hay diferencias en las tres dimensiones competenciales asignadas como más importantes, pero sí en las tres siguientes. El orden para trabajadores con discapacidad intelectual es: 4. $)$ Apariencia personal e higiene; 5. ${ }^{\text {a }}$ ) Gestión y organización; $6 .^{a}$ ) Competencias comunicativas. En el caso de los trabajadores sin discapacidad intelectual el orden varía: $\left.4 .^{a}\right)$ Gestión y organización; 5..$^{a}$ Competencias comunicativas; $6 .^{a}$ ) Apariencia personal e higiene.

La valoración que hacen las empresas colaboradoras es distinta a la realizada por los mediadores laborales, como se puede apreciar en la Tabla 1:

\begin{tabular}{|c|c|c|}
\hline \multicolumn{3}{|c|}{$\begin{array}{l}\text { TABLA 1. Orden de importancia otorgada a las dimensiones competenciales } \\
\text { para la permanencia en el puesto de trabajo }\end{array}$} \\
\hline \multicolumn{2}{|c|}{ Valoración mediadores laborales } & \multirow{2}{*}{$\begin{array}{c}\text { Valoración } \\
\text { empresas colaboradoras }\end{array}$} \\
\hline Trabajadores (sin DI) & Trabajador & \\
\hline $\begin{array}{l}\text { 1. }{ }^{\circ} \text { Responsabilidad, autonomía y } \\
\text { motivación }\end{array}$ & $\begin{array}{r}\text { 1. }{ }^{\circ} \text { Responsa } \\
\text { motivaci }\end{array}$ & $\begin{array}{l}\text { 1. }{ }^{\circ} \text { Responsabilidad, autonomía y } \\
\text { motivación }\end{array}$ \\
\hline 2. ${ }^{\circ}$ Competencias interpersonales & 2. ${ }^{\circ}$ Competencias interpersonales & 2. ${ }^{\circ}$ Gestión y organización \\
\hline $\begin{array}{l}\text { 3. }{ }^{\circ} \text { Flexibilidad, control emocional } \\
\text { y resistencia a la frustración }\end{array}$ & $\begin{array}{l}\text { 3. Flexibilidad, control emocional } \\
\text { y resistencia a la frustración }\end{array}$ & \begin{tabular}{|l} 
3. ${ }^{\circ}$ Flexibilidad, control emocional \\
y resistencia a la frustración
\end{tabular} \\
\hline 4. ${ }^{\circ}$ Gestión y organización & 4. ${ }^{\circ}$ Apariencia personal e higiene & 4. ${ }^{\circ}$ Apariencia personal e higiene \\
\hline 5. ${ }^{\circ}$ Competencias comunica & 5. ${ }^{\circ}$ Gestión y organización & 5. ${ }^{\circ}$ Competencias comunicativas \\
\hline $6 .^{\circ}$ Apariencia personal e higiene & 6. ${ }^{\circ}$ Competencias comunicativas & $6 .^{\circ}$ Competencias interpersonales \\
\hline
\end{tabular}

Fuente: Elaboración propia.

Para las empresas colaboradoras, no hay diferencias en la importancia de las dimensiones competenciales entre trabajadores con y sin discapacidad intelectual.

\section{Ajuste de competencias}

A continuación se expone la distribución de las desviaciones típicas (D. T.) de las respuestas a los ítems en las empresas colaboradoras (Tabla 2):

TABLA 2. Distribución de la D. T. de las respuestas a los ítems en las empresas colaboradoras

\begin{tabular}{|l|l|c|}
\hline \multicolumn{2}{|c|}{ Ítems y dimensión competencial a la que pertenecen } & D. T. \\
\hline G1 & 1. Puntualidad en la hora de entrada al trabajo. & 0.80 \\
\hline G2 & 2. Puntualidad en las horas de salida del trabajo. & 0.97 \\
\hline G3 & 3. Respetar los horarios de descanso, si el puesto contempla estos períodos. & 0.90 \\
\hline G4 & 4. Disposición para acudir, dentro del horario de trabajo, cuando se requiere la presencia del trabajador. & 0.76 \\
\hline G5 & 5. Respetar la planificación temporal de las tareas (realización en el plazo previsto). & 0.72 \\
\hline
\end{tabular}

(C) Ediciones Universidad de Salamanca / CC BY-NC-ND

Siglo Cero, vol. 47 (1), n. ${ }^{\circ}$ 257, 2016, enero-marzo, pp. 37-54

$$
-47-
$$


EVALUACIÓN DE LA METODOLOGÍA EMPLEO CON APOYO (ECA)

EN EL PROGRAMA PROMENTOR (UAM-PRODIS). EL AJUSTE COMPETENCIAL

DOLORES IZUZQUIZA GASSET Y PABLO RODRÍGUEZ HERRERO

\begin{tabular}{|c|c|c|}
\hline \multicolumn{2}{|r|}{ Ítems y dimensión competencial a la que pertenecen } & \multirow{2}{*}{$\begin{array}{l}\text { D. T. } \\
0.51 \\
\end{array}$} \\
\hline G6 & 6. Establecer con autonomía el orden de actividades a realizar cada día. & \\
\hline A1 & 7. Acudir limpio y aseado a su puesto de trabajo. & 0.40 \\
\hline A2 & 8. Dejar todo recogido y ordenado al finalizar el trabajo. & 0.67 \\
\hline F1 & 9. Aceptar de buen grado nuevas tareas. & 0.39 \\
\hline F2 & 10. Saber controlar las emociones cuando algo no sale como espera el trabajador. & 0.63 \\
\hline F3 & 11. Mostrar persistencia ante las dificultades. & 0.56 \\
\hline R1 & 12. Mostrar responsabilidad con las tareas encomendadas. & 0.38 \\
\hline R2 & 13. Implicación con el desarrollo de la empresa. & 0.75 \\
\hline R3 & 14. Mostrar motivación hacia las tareas que debe realizar. & 0.70 \\
\hline R4 & 15. Mostrar interés por realizar las tareas correctamente. & 0.35 \\
\hline R5 & 16. Aportar propuestas respecto a las actividades a realizar (mostrar iniciativa). & 0.89 \\
\hline R6 & 17. Antes de hacer las tareas encomendadas espera que se le ordenen. & 1.01 \\
\hline R7 & 18. Acudir al apoyo natural o al jefe directo para solucionar conflictos con los compañeros. & 0.64 \\
\hline R8 & 19. Solucionar problemas profesionales y de interrelación personal de manera autónoma. & 0.80 \\
\hline R9 & 20. Autonomía en la realización de las tareas. & 0.50 \\
\hline R10 & 21. Buscar la superación personal. & 0.60 \\
\hline R11 & 22. Buscar el reconocimiento del superior para proseguir las tareas. & 0.88 \\
\hline IN1 & 23. Relacionarse adecuadamente con sus superiores. & 0.40 \\
\hline IN2 & 24. Relacionarse adecuadamente con los clientes. & 0.84 \\
\hline IN3 & 25. Saludar al entrar y salir de los espacios compartidos, dar las gracias (agradecer las cosas que recibe). & 0.48 \\
\hline IN4 & 26. Mostrar disposición a estar abierto en sus relaciones. & 0.57 \\
\hline IN5 & 27. Mostrar disposición para ayudar. & 0.49 \\
\hline IN6 & 28. Mostrar disposición a relacionarse con personas o grupos de personas nuevos. & 0.70 \\
\hline IN7 & 29. Adecuar el comportamiento en función de la categoría del interlocutor. & 0.66 \\
\hline $\mathrm{CO} 1$ & 30. Comprender las órdenes orales. & 0.40 \\
\hline $\mathrm{CO} 2$ & 31. Expresarse de forma entendible. & 0.64 \\
\hline $\mathrm{CO} 3$ & 32. Expresar lo que desea oralmente y sin rodeos. & 0.52 \\
\hline $\mathrm{CO} 4$ & 33. Escuchar sin interrumpir. & 0.74 \\
\hline $\mathrm{CO} 5$ & 34. Tener en cuenta las opiniones de los demás. & 0.59 \\
\hline $\mathrm{CO} 6$ & 35. Mantener el contacto visual con el interlocutor. & 0.78 \\
\hline $\mathrm{CO} 7$ & 36. Respetar el turno de palabra. & 0.57 \\
\hline $\mathrm{CO} 8$ & 37. Comprender las órdenes que se le dan por escrito. & 0.65 \\
\hline $\mathrm{CO} 9$ & 38. Escribir de forma legible. & 0.81 \\
\hline $\mathrm{O} 1$ & 39. Orientarse adecuadamente por los diferentes espacios de la empresa. & 0.61 \\
\hline $\mathrm{O} 2$ & 40. Cuando hay que hacer algo fuera de la empresa, orientarse adecuadamente. & 0.90 \\
\hline $\mathrm{O} 3$ & 41. Capacidad para orientarse con un pequeño plano. & 0.94 \\
\hline $\mathrm{O} 4$ & 42. Pedir ayuda si se pierde. & 0.77 \\
\hline it43 & 43. Mantener el ritmo y la carga de trabajo adecuadamente. & 0.40 \\
\hline
\end{tabular}

Nota: $\mathrm{G}$ = Gestión y organización; $\mathrm{A}=$ Apariencia personal e higiene; $\mathrm{F}$ = Flexibilidad, control emocional y resistencia a la frustración; $\mathrm{R}=$ Responsabilidad, autonomía y motivación; $\mathrm{IN}$ = Competencias interpersonales; $\mathrm{CO}=$ Competencias comunicativas; $\mathrm{O}=$ Orientación en la empresa; it43 = añadido sobre desempeño. Fuente: Elaboración propia. 


\section{EVALUACIÓN DE LA METODOLOGÍA EMPLEO CON APOYO (ECA) EN EL PROGRAMA PROMENTOR (UAM-PRODIS). EL AJUSTE COMPETENCIAL DOLORES IZUZQUIZA GASSET Y PABLO RODRÍGUEZ HERRERO}

La Tabla 2 muestra el grado de homogeneidad de las respuestas dadas por las empresas colaboradoras respecto a la importancia que tiene cada competencia o ítem valorado para el desempeño del puesto. Se puede apreciar que las dimensiones más bajas corresponden a los ítems 7, 9, 12, 15, 23, 30 y 43 .

En cualquier caso, debido a que no hay grandes diferencias entre unos valores y otros, se hace necesario metodológicamente realizar un análisis de las respuestas dadas a cada ítem a partir de su distribución de porcentajes. A partir de estos resultados se observa que en prácticamente todos los ítems las mayores tasas de respuesta se producen en las dimensiones competenciales "Responsabilidad, autonomía y motivación” y "Flexibilidad, control emocional y resistencia a la frustración” en cuanto a la importancia conferida para el puesto.

En cada dimensión competencial se ha analizado la puntuación media obtenida tanto para el nivel de competencia del trabajador como para la importancia de la competencia en el puesto. A continuación se describen los resultados más significativos:

- Gestión y organización: Las competencias más importantes son "Establecer con autonomía el orden de actividades...” (3.67 sobre 4) y "Realizar la planificación temporal de las tareas...” (3.60 sobre 4$)$.

- Apariencia personal e higiene: La competencia más valorada es “Acudir limpio y aseado..." (3.81 sobre 4$)$.

- Flexibilidad, control emocional y resistencia a la frustración: La competencia más importante para el puesto es “Aceptar de buen grado nuevas tareas” (3.82 sobre 4).

- Responsabilidad, autonomía y motivación: Las competencias más destacadas son "Mostrar responsabilidad con las tareas encomendadas" (3.88 sobre 4) y "Mostrar interés por realizar las tareas correctamente" (3.86 sobre 4).

- Competencias interpersonales: Se considera la competencia más importante "Relacionarse adecuadamente con sus superiores" (3.80 sobre 4).

- Competencias comunicativas: La competencia más importante es “Comprender las órdenes orales” (3.80 sobre 4).

- Orientación en la empresa: La competencia más destacada es “Orientarse adecuadamente por los diferentes espacios...” (3.66 sobre 4$)$.

- El ítem 43, relativo a "Mantener el ritmo y la carga de trabajo adecuadamente", es uno de los más valorados con un 3.80 sobre 4.

En cuanto a la comparación entre la valoración del equipo de mediadores laborales y las empresas colaboradoras, en las puntuaciones finales más bajas prácticamente no existe diferencia entre los distintos criterios de ponderación, mientras que en las puntuaciones finales más altas, dicha diferencia es algo menor de 10 puntos.

En términos psicométricos la escala compuesta por los 43 ítems presenta un buen comportamiento, con un valor alfa de Cronbach (nivel de competencia del trabajador) 
$=0.865$ y un valor alfa de Cronbach (importancia de la competencia para el puesto) $=0.913$. Estos resultados deben tomarse con cautela ya que, si bien los valores de alfa son bastante elevados, con el tamaño muestral disponible no resulta adecuado hablar estrictamente de fiabilidad de la consistencia interna de la escala hasta realizar nuevas aplicaciones con muestras mayores.

Lo que sí indican los niveles de alfa encontrados es que las puntuaciones finales altas se corresponden con puntuaciones altas en la mayoría de los ítems, lo mismo que las puntuaciones final-ítems intermedias y las bajas. En cuanto a la validez de contenido, los resultados indican un elevado acuerdo en el orden de importancia conferido a las diferentes competencias. Además, se identifican contenidos importantes para el desempeño en el puesto en todas las dimensiones evaluadas, lo que resulta positivo desde el punto de vista de su representatividad y relevancia. En conclusión, puede decirse que la escala de evaluación utilizada muestra un grado de fiabilidad suficiente y permite realizar una aproximación al ámbito de estudio, lo que invita a su aplicación en nuevas muestras y en futuras investigaciones.

Por último, otro resultado a destacar es que la valoración del nivel competencial de cada trabajador guarda relación con la importancia conferida a las competencias evaluadas para el desempeño del puesto. Cuanto más importantes son las competencias evaluadas para el desempeño del puesto existe una tendencia a valorar más positivamente el nivel competencial del trabajador que desempeña dicho puesto.

\section{Discusión}

A partir de estos resultados, se pueden obtener conclusiones de interés para la mejora en el proceso de ECA, especialmente en relación al ajuste competencial necesario para mantener los puestos de trabajo.

En primer lugar, se confirma la importancia de la figura del mediador laboral, al igual que en otras investigaciones (Arenas y González, 2010; Bennett y cols., 2010; Egido, Cerrillo y Camina, 2009; Goh y Bambara, 2013; Izuzquiza y Herrán, 2010; O’Brien y Callahan, 2010). Se ha demostrado que el mediador laboral no solo ejerce un papel esencial al inicio de la inserción laboral, sino también en el mantenimiento en el puesto de trabajo: más del 90\% de los informantes encuestados considera importante que los mediadores laborales continúen visitando la empresa incluso cuando el trabajador ha alcanzado la autonomía suficiente en las tareas que se le han encomendado. La frecuencia de las visitas de seguimiento del mediador laboral debería ser, según la mayoría de las empresas, de una vez al mes. Se asume, por tanto, que el objetivo del ECA es que el trabajador pueda desempeñar sus tareas de forma autónoma.

Considerando los resultados obtenidos, los mediadores laborales tendrían que mantener en el tiempo apoyos en las siguientes áreas:

- Aspectos tangibles: orden de actividades diario, realizar las tareas en el plazo previsto, aceptar de buen grado tareas nuevas, aportar propuestas ante las tareas a realizar, mostrar iniciativa, adecuar el comportamiento en función de la 
categoría del interlocutor, relacionarse adecuadamente con los clientes, comprender las órdenes que se reciben por escrito, expresar lo que se desea sin rodeos, expresarse de forma entendible y comprender las órdenes orales.

- Aspectos intangibles: mostrar persistencia ante las dificultades, saber controlar las emociones cuando algo no sale como espera el trabajador, buscar la superación personal y tener en cuenta opiniones de los demás.

De lo anterior se desprende que la presencia del mediador laboral constituye un primer factor importante para el mantenimiento en el puesto. Si bien para la inserción laboral se necesita de una buena adaptación de los puestos de trabajo, para el mantenimiento en el puesto se necesita de un ajuste y reajuste competencial entre el trabajador con discapacidad intelectual y el puesto que sea continuado en el tiempo.

El análisis de las dimensiones competenciales permite detectar qué competencias son las más valoradas por las empresas colaboradoras y por los mediadores laborales, lo cual puede ayudar a definir los planes de formación individualizados que se diseñan para mejorar la empleabilidad. En este sentido, es pertinente diseñar planes de formación inicial y permanente que se adapten a las necesidades percibidas, dando un mayor peso a las dimensiones competenciales más valoradas.

Existe un acuerdo generalizado entre los mediadores laborales y las empresas colaboradoras en otorgar a la dimensión competencial "Responsabilidad, autonomía y motivación” el primer puesto en importancia para el mantenimiento en el puesto. Esta dimensión es fundamental para el desempeño de un puesto de trabajo continuado en el tiempo, no solo para los trabajadores con discapacidad. Es el aspecto que más se puede llegar a valorar en un trabajador como valor para la empresa, más allá de conocimientos técnicos y otro tipo de habilidades.

Desde el punto de vista de apoyo laboral, más que una dimensión competencial se puede entender la responsabilidad, la autonomía y la motivación como un fin o una meta a alcanzar en el desarrollo personal de las personas con discapacidad intelectual, en este caso a través del trabajo remunerado y normalizado. Desde el punto de vista empresarial, esta dimensión competencial tendrá (en principio) un mayor componente práctico, relacionado con la capacidad del trabajador para asumir tareas de forma eficiente. En este sentido, no parece casual que la dimensión elegida en segundo lugar de importancia por los mediadores laborales sea las "Competencias interpersonales", mientras que la elegida en el mismo puesto por las empresas colaboradoras sea "Gestión y organización".

Sobre la adaptación de los puestos de trabajo, es reseñable que los informantes consideran que resulta accesible y viable desde un punto de vista práctico. Las empresas colaboradoras valoran positivamente la intervención de los mediadores laborales en estas adaptaciones. Además, es importante resaltar que para los informantes los puestos de trabajo evaluados resultan importantes para la empresa, dentro de su nivel de jerarquía, lo que refleja una apuesta seria por parte de las empresas colaboradoras por estos trabajadores. Teniendo en cuenta que más del $60 \%$ de los puestos se crearon cuando se incorporó el trabajador, estos datos reflejan lo que las personas con discapacidad intelectual pueden aportar a las empresas (Coutts y Riddell, 2013). 


\section{EVALUACIÓN DE LA METODOLOGÍA EMPLEO CON APOYO (ECA) EN EL PROGRAMA PROMENTOR (UAM-PRODIS). EL AJUSTE COMPETENCIAL DOLORES IZUZQUIZA GASSET Y PABLO RODRÍGUEZ HERRERO}

En conjunto, estos aspectos permiten hacer una valoración positiva del Programa Promentor y del Servicio de Inclusión Laboral de la Fundación Prodis, en términos de pertinencia, coherencia, cobertura y eficacia. En cualquier caso, los resultados obtenidos pueden dar lugar a reflexiones que mejoren el apoyo laboral que se da a los trabajadores.

La investigación que se ha llevado a cabo tiene unas limitaciones que hacen necesaria la realización de futuros estudios. La complejidad y el grado de profundidad de las categorías de análisis hacen que un enfoque cuantitativo sea insuficiente y que, por consiguiente, se requieran estudios posteriores de carácter cualitativo, que profundicen en los factores que intervienen en el ajuste competencial y el mantenimiento del puesto de trabajo. Así, se recomienda para una futura investigación la realización de entrevistas a las empresas colaboradoras, especialmente a las figuras de responsable/jefe y apoyos naturales. Estas entrevistas permitirán realizar una mejor triangulación de los datos.

Por otra parte, es necesario realizar investigaciones cercanas en otros ámbitos territoriales a nivel nacional e internacional, para desarrollar un análisis comparativo que permita definir factores transversales que influyen en la permanencia en el puesto de trabajo. Especialmente relevante es el desarrollo de investigaciones internacionales en las que puedan participar equipos que investiguen en sus circunstancias contextuales acerca de la mejora de la metodología de ECA. La promoción de investigaciones internacionales puede resultar en aportaciones sustantivas al modelo.

Estas líneas de investigación pueden contribuir a la mejora de la metodología de ECA desde una práctica basada en evidencias. El horizonte es que las personas con discapacidad intelectual puedan disfrutar de los derechos de cualquier ciudadano y, por consiguiente, participar activamente en la construcción de una sociedad mejor.

\section{Referencias bibliográficas}

Arenas, M. L. y GonzÁlez, R. (2010). El preparador laboral en el empleo con apoyo. Educación y Futuro, 23, 43-50.

Becker, D. R., Xie, H., McHugo, G. J., Halliday, J. y Martínez, R. A. (2006). What predicts supported employment program outcomes? Community Mental Health Journal, 42 (3), 303-313.

Bennett, K., Brady, M., Scott, J., Dukes, C. y Frain, M. (2010). The effects of covert audio coaching on the job performance of supported employees. Focus on Autism and Other Developmental Disabilities, 25 (3), 173-185.

Bisquerra, R. (2004). Metodología de investigación educativa. Madrid: La Muralla.

Bond, G. R., Peterson, A. E., Becker, D. R. y Drake, R. E. (1997). Validation of the Revised Individual Placement and Supported Fidelity Scale (IPS-25). Psychiatric Services, 63 (8), 758-763.

Cerrillo, R., Izuzquiza, D. y Egido, I. (2013). Inclusión de jóvenes con discapacidad intelectual en la Universidad. Revista de Investigación en Educación, 11 (1), 41-57.

CouTTS, P. y Riddell, S. (2013). Employer's views of supported employment for disabled people in Scotland. Edimburgh: The University of Edinburgh. 
EVALUACIÓN DE LA METODOLOGÍA EMPLEO CON APOYO (ECA)

EN EL PROGRAMA PROMENTOR (UAM-PRODIS). EL AJUSTE COMPETENCIAL

DOLORES IZUZQUIZA GASSET Y PABLO RODRÍGUEZ HERRERO

Egido, I., Cerrillo, R. y Camina, A. (2009). La inclusión social y laboral de las personas con discapacidad intelectual mediante los programas de empleo con apoyo. Un reto para la orientación. REOP, 20 (2), 135-146.

Esteban, B. y Jordán de UrRíes, B. (2006). Empleo con apoyo para personas con discapacidad intelectual y para personas con enfermedad mental. Comparación metodológica en dos proyectos piloto. Siglo Cero. Revista Española sobre Discapacidad Intelectual, 37 (2), 63-78.

European Comission (2011). Supported employment for people with disabilities in the UE and EFTA-EEA. Luxembourg: Publications Office of the European Union.

Evans, M., Lopes, I., Katsouda, C., Diepeveen, A., Fleming, M., Haddock, M., Hazzard, T., Schuller, S., Johnova, M., Boxava, P., Martínez, S., Jordán de Urríes, B., Eklund, B., Sariola, L. y Haltunen, H. (2005). Information Booklet and Quality Standars. Belfast: European Union of Supported Employment.

Flores, N., Jenaro, C., Orgaz, M. y Martín, M. (2011). Understanding quality of working life of workers with intellectual disabilities. Journal of Applied Research in Intellectual Disabilities, 24 (2), 133-141.

Foley, K.-R., Girdler, S, Downs, J., Jacoby, P., Bourke, J., Lennox, N., Einfeld, S., Llewellyn, G., Parmenter, T. R. y Leonard, H. (2014). Relationship between family quality of life and day occupations of young people with Down syndrome. Soc Psychiatry Psychiatr Epidemiol, 49, 1455-1465.

GoH, A. y BAmBARA, L. (2013). Video self-modeling: a job skills intervention with individuals with intellectual disability in employment settings. Education and Training in Autism and Developmental Disabilities, 48 (1), 103-119.

Hall, A., Bose, J., Winsor, J. y Migliore, A. (2014). Knowledge translation in job development: strategies for involving families. Journal of Applied Research in Intellectual Disabilites, 27 (5), 489-492.

IzUZquiza, D. (2012). El valor de la inclusión educativa de jóvenes con discapacidad intelectual en las instituciones de Educación Superior: el Programa Promentor. Bordón, 64 (1), 109-126.

Izuzquiza, D. y Herrán, A. de la (Coords.) (2010). Discapacidad intelectual en la empresa. Las claves del éxito. Madrid: Pirámide.

Jordán DE URRíes, B. (2010). Empleo con apoyo. Educación y Futuro, 23, 31-42.

Jordán de Urríes, B., Beyer, S. y Verdugo, M. Á. (2008). Un estudio comparativo del empleo con apoyo en Europa. En II Premio de Investigación sobre Discapacidad Intelectual. Manresa: AMPANS.

O’Brien, J. y Callahan, M. (2010). Employment support as knowledge creation. Research and Practice for Persons with Severe Disabilities, 35 (1-2), 31-38.

Pérez, C. e IzuzQuiza, D. (2011). Diseño y validación de un cuestionario para valorar el ajuste competencial de trabajadores con discapacidad intelectual en las empresas. Trabajo Final de Máster, Universidad Autónoma de Madrid.

Santamaría, M., Verdugo, M. Á., Orgaz, B., Gómez, L. E. y Jordán de Urríes, F. B. (2012). Calidad de vida percibida por trabajadores con discapacidad intelectual en empleo ordinario. Siglo Cero. Revista Española sobre Discapacidad Intelectual, 43 (2), 46-61.

Schalock, R., Borthwick-Duffy, S., Bradley, V., Buntinx, W., Coultier, D., Craig, E., Gómez, S., Lachapelle, Y., Luckasson, R., Reeve, A., Shogren, K., Snell, M., Spreat, S., Tassé, M., Thompson, J., Verdugo, M. Á., Wehmeyer, M. y Yeager, M. (2010). Intellectual disability: Definition, classification, and systems of supports. $11^{\text {th }}$ Edition of the AAIDD Definition Manual. Washington, DC: American Association on Intellectual and Developmental Disabilities. 
Stufflebeam, D. L. (2003). The CIPP model for evaluation. New Directions for Program Evaluation, 19, 7-98.

Substance Abuse and Mental Health Services Administration (2009). Supported Employment: the Evidence. Rockville, MD: Center for Mental Health Services, Substance Abuse and Mental Health Services Administration, U.S. Department of Health and Human Services.

Verdugo, M. Á., Benito, M. C., Orgaz, B., Jordán de Urríes, F. B., Martín-Ingelmo, R. y SAntamaría, M. (2012). Influencia de un programa de empleo con apoyo en la calidad de vida percibida por sus usuarios. Siglo Cero. Revista Española sobre Discapacidad Intelectual, 43 (3), 69-83.

Verdugo, M. Á., Jordán de Urríes, F. B., Martín, R., Vicente, C. y Sánchez, M. C. (2009). Impact on quality of life and self-determination of a national program for increasing supported employment in Europe. Journal of Vocational Rebabilitation, 31 (1), 55-64.

Verdugo, M. Á., Jordán de Urríes, B. y Vicent, C. (2009). A Multicomponent Evaluation System of Supported Employment Programs. Journal of Vocational Rehabilitation, 31 (1), 65-72.

Wehman, P., Moon, S., Everson, J. M., Wood, M. y Barcus, J. M. (1987). Transition from school to work. New challenges for youth with severe disabilities. Baltimore, Maryland: Paul H. Brookes.

West, E. y Patton, H. (2010). Positive behavior support and supported employment for adults with severe disability. Journal of Intellectual \& Developmental Disability, 35 (2), 104-111.

World Medical Association Declaration of Helsinki (2002). Declaration of Helsinki: Ethical principles for medical research involving human subjects. JAMA, 284, 3043-3045. 


\section{RESEÑAS}

Flórez, J., Garvía, B. y Fernández, R. (2015). Sindrome de Down: neurobiología, neuropsicologia, salud mental. Bases para la intervención en el aprendizaje, la conducta $y$ el bienestar mental. Madrid: CEPE, Fundación Iberoamericana Down21. ISBN 97884-7869-999-5, 527 pp.

Este tratado, elaborado por Jesús Flórez, Beatriz Garvía y Roser Fernández-Oloria y coeditado por la Fundación Iberoamericana Down21 y la editorial CEPE, quiere aportar una visión actualizada de la perspectiva de la neurociencia y la psicología en relación con el síndrome de Down, además de exponer los fundamentos para actuar y las grandes posibilidades de acción para influir sobre la cognición, la conducta y la salud mental.

La trayectoria profesional y las experiencias personales de los autores garantizan esta obra en la que se nos acerca a la realidad del síndrome de Down, con sus situaciones y condiciones positivas y negativas, con la esperanza de derribar los perjuicios sociales que han levantado barreras y creyendo en la capacidad de las personas con síndrome de Down.

A lo largo de 19 capítulos se encuentran investigaciones sobre genética, neurología, psicología, vida adulta, envejecimiento, problemas conductuales, problemas mentales, atención, memoria, lenguaje... que pretenden responder, basándose en el conocimiento de la neurociencia y la psicología, a cuáles son los rasgos comunes de las personas con síndrome de Down siendo conocedores de las diferencias existentes entre los seres humanos. La presencia de un cromosoma 21 extra, con sus más de 500 genes y otro material genético propio de ese cromosoma, introduce una modificación con consecuencias reales sobre la constitución del organismo humano expresada en sus múltiples órganos y sistemas.

Este libro trata de abordar de forma global lo que el cerebro es y significa para poder modificar y realizar una intervención tanto en el aprendizaje como en la conducta y en la salud mental, siendo este triple análisis la novedad de que aporta esta obra.

La primera parte del libro se centra en la realidad del cerebro, su desarrollo y genética, así como la organización de redes neuronales y las consecuencias que produce la trisomía del cromosoma 21, su evolución, cambios o mejora de posibilidades.

En la segunda parte se abordan los temas fundamentales de las funciones cerebrales estudiados por la moderna neuropsicología. En los últimos capítulos se ofrece una visión sobre el bienestar, la construcción de la identidad y la salud mental, deteniéndose en cuáles son los principales trastornos mentales y sus tratamientos psicoterapéuticos y farmacológicos, para apreciar los logros actuales y los futuros retos.

La realidad que se aprecia en las personas con síndrome de Down a lo largo de su vida, la calidad de vida alcanzada en función de sus características personales, de la riqueza de los apoyos recibidos y de los propios esfuerzos supone una apertura de oportunidades para todas las personas. La sociedad no es consciente de la importancia de cambiar actitudes para enriquecer el propio conocimiento, la diferencia es un elemento esencial en los seres humanos por lo que el rechazo que en ocasiones sufren las personas con síndrome de Down es muy probable debido a un desconocimiento del cúmulo de posibilidades de las personas con síndrome de Down. 


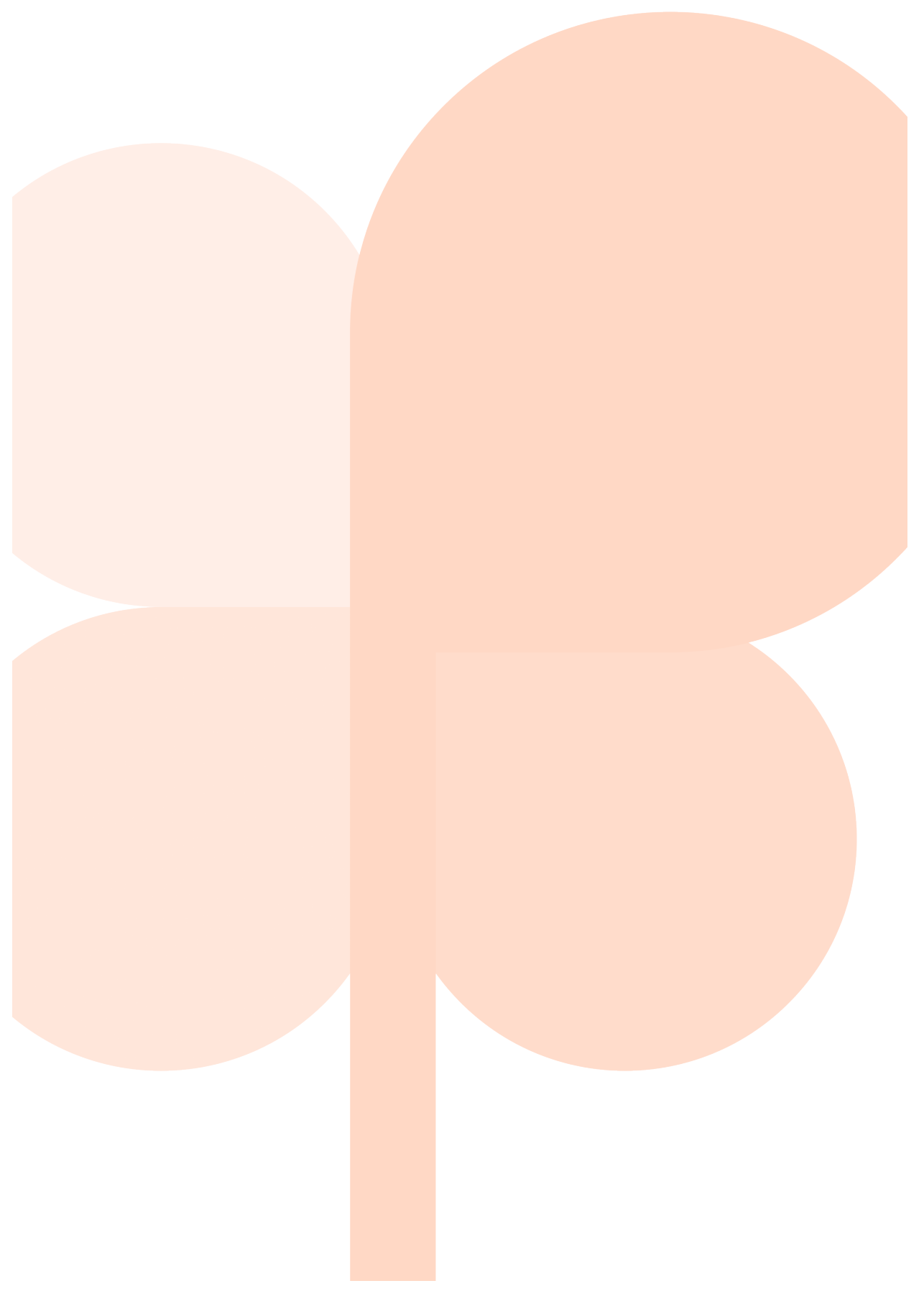

